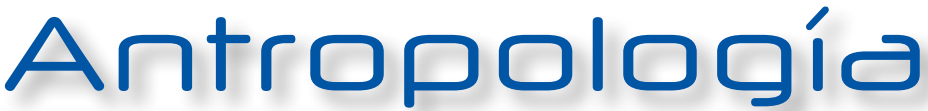

\section{Efectos de la tecnología en los cuidados de enfermería}

\section{Efeitos da tecnologia na assistência da enfermagem Effects of technology in nursing care}

Karina Jazmín Marrujo Pérez ${ }^{1}$ y Domingo Palacios Ceña ${ }^{2}$

${ }^{1}$ Licenciada en Enfermería, Estudiante de Maestría Profesionalizante de Enfermería de la universidad Autónoma de Chihuahua. Karila_192@hotmail.com

${ }^{2}$ Doctor en Ciencias de la Salud. Universidad Rey Juan Carlos.domingo.palacios@urjc.es

Cómo citar este artículo en edición digital: Marrujo Pérez, K.J., y Palacios Ceña, D. (2016). Efectos de la tecnología en los cuidados de enfermería. Cultura de los Cuidados (Edición digital), 20( 46). Disponible en: < http://dx.doi.org/10.14198/cuid.2016.46.12>

Correspondencia: Domingo Palacios Ceña. Despacho 1056, departamental II, Facultad Ciencias Salud. Universidad Rey Juan Carlos. Avenida Atenas s/n. 28922, Alcorcon, Madrid, España. Tel: +34.91.488.8883

Correo electrónico: domingo.palacios@urjc.es

Recibido: 14/10/2015; Aceptado: 20/07/2016

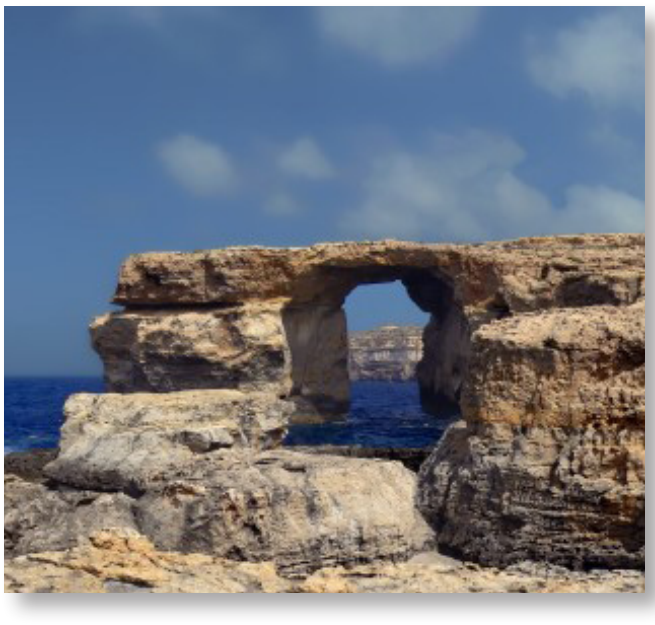

ABSTRACT

The vision of technology as an aid to improve the conditions of those influences multiple aspects of health and care. Technology in nursing and care covered; the role of the nurse as a link between technology and the patient, the application of an ethic of care, training of management of technology and education in various fields. Lack of management with performance problems can cause anxiety and stress on nurses, the use of technology within the training of nurses, reinforces the skills with which it comes to clinical practice or institutions, promoting adaptation to the workplace. The innovate depends not only on health institutions, implementation of new technologies, seeks that professionals see an opportunity for professional growth and is interested in the development of projects that are aimed to use and implementation. The technology is a neutral element, are the actions of the nurse which determine the presence of positive or negative associated with the application of technology aspect.

Key words (DeCS): Education Nursing; Nursing Care; Nursing Informatics; Biomedical Technology; Technology.

\section{RESUMO}

A visão da tecnologia como uma ferramenta que auxilia a melhora das condições dos sujeitos influencia em múltiplos aspectos da atenção à saúde e dos cuidados. Tecnologia em enfermagem e no cuidado engloba o papel do enfermeiro como elo de ligação entre a tecnologia e o paciente, a aplicação de uma ética do cuidado e a formação em gestão de tecnologia 
e educação em distintos campos. A inabilidade de gestão relacionada aos problemas de desempenho tecnológicos podem causar ansiedade e estresse nos enfermeiros. A utilização de tecnologia no âmbito da formação de enfermeiros reforça as habilidades com as quais o profissional irá deparar-se na prática clínica ou instituições, favorecendo uma melhor adaptação ao local de trabalho. O inovar depende não só nas instituições de saúde, mas também da implementação de novas tecnologias, da busca por parte dos profissionais que veem uma oportunidade de crescimento profissional e se interessem no desenvolvimento de projetos que visam a utilização e implementação das mesmas. A tecnologia é um elemento neutro, são as ações do enfermeiro que determinarão a presença de positivo ou negativo associado com a aplicação da mesma.

Palavras chave (DeCS): Educação em Enfermagem; Cuidados de Enfermagem; Informática em Enfermagem; Tecnologia Biomédica; Tecnologia.

\section{RESUMEN}

La visión de la tecnología como herramienta de ayuda destinada a mejorar las condiciones de las personas influye en múltiples aspectos de la asistencia y del cuidado. La tecnología en enfermería y en los cuidados abarca; el papel de la enfermera como nexo de unión entre la tecnología y el paciente, la aplicación de una ética del cuidado, la capacitación del manejo de la tecnología y la educación en distintos ámbitos. La falta de manejo junto a problemas de funcionamiento pueden provocar ansiedad y estrés en las enfermeras, el uso de la tecnología dentro de la formación de profesionales de enfermería, refuerza las habilidades con las que se llegue a la práctica clínica o a las instituciones, favoreciendo la adaptación al entorno laboral. El innovar no solo depende de las instituciones de salud, la implementación de las nuevas tecnologías, busca que los profesionales vean una oportunidad para el crecimiento profesional y que se interesen por el desarrollo de proyectos que vayan encaminadas al uso y su aplicación. La tecnología es un elemento neutro, son las actuaciones de la enfermera lo que determina la presencia de aspecto positivos o negativos asociados a la aplicación de tecnología.

Palabras clave: Educación en Enfermería, Atención de Enfermería, Informática Aplicada a la Enfermería, Tecnología Biomédica, Tecnología.

\section{INTRODUCCIÓN}

En la última década las inversiones y el gasto asociado a la adquisición de tecnologías sanitarias ha llegado a alcanzar el 8.2\% del gasto destinado en Sanidad en España. El incremento de su implantación en los distintos entornos sanitarios provoca que los profesionales sanitarios deban adaptarse a las nuevas herramientas y técnicas que van apareciendo, las cuales cada vez más influyen en su trabajo y en sus disciplinas. La visión de la tecnología como herramienta de ayuda destinada a mejorar las condiciones de las personas sigue influyendo en múltiples aspectos de la asistencia y del cuidado.

En el presente trabajo se mostrarán los efectos de la tecnología en la aplicación de cuidados y el papel de la enfermera como disciplina frente al avance progresivo de la tecnificación del entorno donde desarrolla su actividad.

A modo de ejemplo dentro de estos equipamientos tecnológicos se encuentran la ventilación mecánica invasiva, ventilación mecánica no invasiva con ventiladores tipo cpap/ bipap, 
los monitores de diálisis y de hemofiltración, desfibriladores, etc.

\section{DESCRIPCIÓN}

La influencia de la tecnología en la enfermería y en los cuidados abarca distintos puntos entre los que se encuentran; el papel de la enfermera como nexo de unión entre la tecnología y el paciente, la aplicación de una ética del cuidado, la capacitación del manejo de la tecnología y la educación en distintos ámbitos.

\section{Enfermería; nexo de unión entre la tecnolo-} gía y el paciente.

La consideración de la enfermera como unión entre un entorno tecnificado y el contacto humano con el paciente es una visión y construcción que se a asociado a la enfermería desde el comienzo de manejo de equipamiento tecnológico complejo en la asistencia (Arredondo González, y Siles González, 2009). Incluso dentro de la propia enfermería se ha considerado como tal (Barnard y Sandelowski, 2001). Autores como Barnard y Sandelowski (2001) muestran como este papel que la enfermería adopta esta asociado a la construcción social del concepto de tecnología. Es la construcción de su significado lo que determina un comportamiento determinado por las enfermeras respecto a su papel en relación a la tecnología y al manejarla. La clave esta en la orientación que se de al aplicar esa tecnología, ya que por si misma es un elemento neutro, es como la utilice lo que le dará el carácter positivo o negativo derivado de su aplicación (Escudero, 2003; Feito, 2005; Dragon, 2006). El significado de la tecnología reside en considerarla como una herramienta que ayuda a cubrir las necesidades y problemas de la persona enferma y no del profesional. Su significado se construye en el momento que se ayuda al paciente a comprender su experiencia de enfermar mediante la aplicación de técnicas y tecnologías durante su proceso, le permiten establecer un antes y un después y sobre todo la necesidad de utilizar medios (en este caso tecnológicos) para ayudarle a superar la situación de enfermedad. Otros autores como Arredondo González y Siles González (2009), destacan que la tecnología en sí misma, no es el único factor que produce deshumanización, este proceso asociado a la tecnología es multifactorial.

\section{La ética del cuidado.}

Es una ética en la que se reflexiona sobre los aspectos de la persona, no solo los físicos, sino también los emocionales, vivénciales, valores y de reflexión, como indica Feito Grande :"...todos ellos elementos que componen la vida moral de las personas, pero que no tienen cabida en un modelo de fundamentación racional de corte lógico y deductivista" (Feito, 2005). El riesgo que conlleva la aplicación de medios tecnológicos en el cuidado por parte de la enfermería es la pérdida de la visión integral del individuo y dar más importancia a la máquina que a la persona (Haghenbeck, 2005). Está pérdida de globalidad se debe a que la enfermera maneja la tecnología, considerando a la persona como un objeto y no como un sujeto. De esta manera la persona es entendida como una "entidad" que porta una enfermedad o proceso al que se estudia, vigila y trata. Por otro lado al percibirla como un sujeto se acepta la existencia de una relación de ayuda enfermera- paciente, se le considera como participante en la aplicación del cuidado ayudado por la tecnología (Marden, 2005; Moore y Wasson, 2006). Mientras que en la consideración sujeto de la persona se potencia y mantiene la autonomía, en la objeto, se pri- 
ma la beneficencia de los procedimientos siendo el resto de visiones un factor secundario al objetivo primario que es la curación.

\section{Capacitación en el manejo de tecnología.}

Como todo procedimiento la tecnología debe ser enseñada y precisa de una formación específica para su manejo y aplicación por dos razones:

- La capacitación y especialización de las enfermeras se asocia a un descenso de la mortalidad y a un aumento de la detección de complicaciones potencialmente reversibles asociadas a tratamientos y técnicas, entre ellas las quirúrgicas (Needleman, et al., 2002; Airen, et al., 2011).

- La falta de formación en su manejo junto a problemas de funcionamiento pueden provocar ansiedad y estrés en las enfermeras, errores en la detección de alarmas, en la valoración del estado del enfermo respecto a las lecturas del equipamiento y la falta de criterios para detectar precozmente problemas asociados a su manejo (Barnard y Sandelowski, 2001) .

La formación sobre el equipamiento tecnológico no debe ser solo respecto a su aplicación, también respecto a la detección de anomalías durante su funcionamiento que podrían provocar daños en el paciente. Al menos a un nivel básico para mantener la seguridad del paciente y del profesional independientemente que el equipo sea revisado por personal técnico de mantenimiento o similares.

Autores como Haghenbeck (2005) afirman que el empleo de tecnología supone para las enfermeras un proceso de adaptación que conlleva sentimientos y sensaciones de falta de autoestima, estrés e incertidumbre. La integración de la tecnología debe de ser paralela a la formación y debería ser incluida en los planes educativos del pre y postgrado. Los contenidos teóricos junto a la adquisición de destrezas y habilidades en su funcionamiento y manejo ante complicaciones e imprevistos debería ser un punto a desarrollar en los talleres formativos (Ansaloni, 2007).

Otro aspecto es la presencia de problemas de tipo ético y moral en varios aspectos de la aplicación de equipamiento tecnológico, el primero aparece al asignar a una enfermera sin formación específica el manejo de una tecnología que pude provocar daños al paciente (la ventilación mecánica por ejemplo) y el segundo al no aceptar en determinadas instituciones o servicios a pacientes que portan un determinado equipamiento tecnológico por falta de personal cualificado para su manejo. Como muestra Mahon (2006) en el caso de pacientes terminales que precisan de ingreso en centros paliativos (hospice) y por necesitar determinadas tecnologías para sus procesos (ventilación mecánica, diálisis...), pero que no son la causa principal de su estado terminal, se les deniega el acceso.

\section{Educación sanitaria.}

Está dirigida no sólo al paciente, también a la familia y al cuidador principal, estos últimos destacan sobre todo en el manejo de tecnologías en niños. Es vital crear programas específicos de formación y preparación antes del alta (Tearl y Hertzog, 2007) para que al llegar al domicilio el paciente y la familia puedan manejar cualquier situación que surja derivada del equipamiento tecnológico, como es el caso de ventiladores, diálisis peritoneal, oxigenoterapia, etc. Una complicación propia del uso de tecnología en el domicilio es la presencia de situaciones denominadas quemamiento por el tratamiento, "burden of treatment", en los que la persona presenta diversas sintomatologías y complicaciones derivadas del uso de la tecno- 
logía, no físicas, sino psicológicas y de adaptación a su empleo (Jordan, et al., 2006). Johansson, et al. (2005) describen entre otras estrés y ansiedad. No es suficiente con prescribir una terapia y aplicar una tecnología determinada (Sneed, et al., 2004) es necesario la creación de programas específicos en función del tipo de equipamiento tecnológico utilizado (Ingadottir y Jonsdottir, 2006).

La aplicación de una tecnología en el domicilio debe estar acompañada de sesiones formativas de educación y de un sistema de apoyo para facilitar su manejo y evitar la ansiedad asociada a la responsabilidad del manejo de la tecnología.

\section{Educación y formación de la enfermera.}

La educación en enfermería cada día está cambiando y este cambio se puede ver con la aplicación de nuevas tecnologías desde la enseñanza (Hallmark, 2015). El uso de la tecnología dentro de la formación de profesionales de enfermería, puede reforzar las habilidades con las que se llegue a la práctica clínica o a las instituciones, favoreciendo la adaptación al área o cuando se comience la vida laboral (Brault, et al., 2015). Cada vez son más las universidades que desarrollan programas donde los estudiantes tengan una experiencia de simulación clínica, que se asemeje a la realidad que se vive en los hospitales, con ello se favorece la formación tanto de docentes y alumnos, logrando un lenguaje común dentro del ámbito clínico y académico (Lily, Fitzpatrick y Madigan, 2015).

Aun así y pudiendo observar las ventajas que implica el poder llevar estas tecnologías desde el ámbito académico existen limitaciones que impiden su aplicación y desarrollo, como: a) profesionales poco cualificados, con deficiencias o pocas habilidades para enseñar;

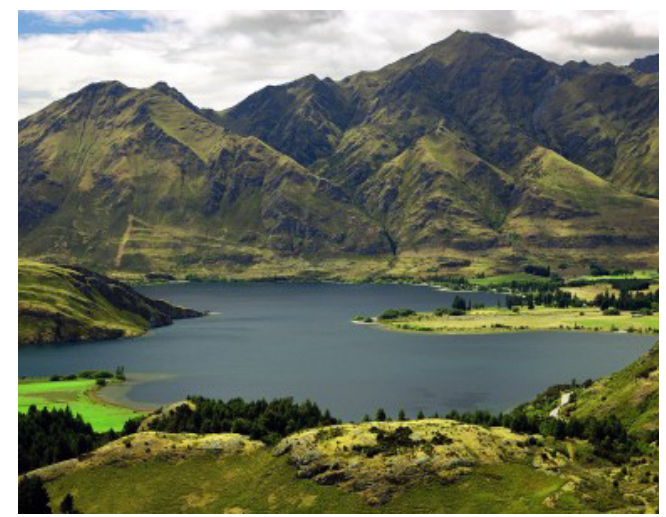

b) la falta de tiempo o de interés por quererlas aplicar; c) falta de implicación de las instituciones sanitarias y/o educativas por desconocimiento, de que existen y poder aplicarlas desde la formación (Krau, 2015; Lily, Fitzpatrick y Madigan, 2015). Es importante que se promueva la aplicación de estas tecnologías desde la enseñanza con esto se facilitaría la comunicación de todos los profesionales que las utilizan y ofrecería oportunidades para mejorar el cuidado del paciente (Brault, et al., 2015).

\section{Aplicación, innovación y desarrollo de la tecnología en enfermería.}

La aplicación de nuevas tecnologías en las áreas de trabajo de los profesionales de enfermería, es parte de los retos del futuro pero no solo su uso y aplicación, es importante que los sistemas tecnológicos que se quieran implementar cumplan con las necesidades que la profesión requiere y cuales son más aplicables a las características y conocimientos de la practica en enfermería, respetando los criterios de calidad y mejorando el cuidado otorgado a los pacientes (Brown, 2015).

Esto se encuentra relacionado con la innovación, buscando la creación de nuevos programas que también se adapten a la modernidad y a las nuevas tecnologías que van saliendo al mercado y no siempre tienen que ver en la 
práctica directa, pero si se pueden adaptar para que esta sea aún más fácil, como lo es la utilización de las redes móviles. Estas redes son una plataforma eficaz para la comunicación y para el acceso a información de aplicación en los cuidados y la salud, como control de patologías crónicas, resolución de dudas, aplicación de cuidados en zonas remotas, etc (Becker, et al., 2015; Ketel, 2015).

El innovar no solo depende de las instituciones de salud, la implementación de estas nuevas tecnologías, también busca que los profesionales de enfermería aprendan y respondan a estas nuevas aplicaciones, que vean una oportunidad para el crecimiento profesional y que se interesen por el desarrollo de proyectos que vayan encaminadas al uso y aplicación de estas, como la reducción de los reingresos hospitalarios, innovando la práctica de enfermería más allá de un ámbito institucional (Bastos, 2002; Farrar, 2015).

\section{CONCLUSIONES}

La tecnología es un elemento neutro, son las actuaciones de la enfermera lo que determina la presencia de aspecto positivos o negativos asociados a la aplicación de tecnología. La formación es vital para construir el significado de la enfermera y su relación con la tecnología y ayudar a percibir a la persona como un sujeto autónomo, no como un objeto al que aplicamos una máquina.

La aplicación de esta tecnología en medios como el domicilio y otros centros debe ser evaluada por el médico, la enfermera, el paciente y sobre todo por la familia o el cuidador principal encargada de la persona enferma. El motivo es claro, la tecnología ofrece beneficios en el proceso de la enfermedad pero lleva consigo una gran carga de incertidumbre, ansiedad y estrés relacionado con su manejo y con la res- ponsabilidad de poder provocar un daño involuntariamente por falta de conocimiento $y$ habilidad.

\section{BIBLIOGRAFÍA}

- Airen, L., Clarke, S., Sloane, D., Sochalski, J., y Silber, J. (2002). Hospital nurse staffing and patient mortality, nurse burnout, and job dissatisfaction. Journal of the American Medical Association Oncology, 288(16), 19871993.

- Ansaloni, P., 2007. Role of nurses in managing complex instruments. Giornale Italiano di Cardiologia, 8(5), 4245.

- Arredondo González, C.P., y Siles González, J. (2009). Tecnología y Humanización de los Cuidados. Una mirada desde la Teoría de las Relaciones Interpersonales. Index de Enfermería, 18(1), 32-36.

- Barnard, A., y Sandelowski, M. (2001). Technology and humane nursing care: (ir) reconcilable or invented difference? Journal of Advanced Nursing, 34(3), 367-375.

- Bastos, M., (2002). Saber e a tecnología: mitos de um centro de tratamiento intensivo. Rev Latino-Am Enfermagem, 10(2), 131-136.

- Becker, M., Behrends, M., Barthel, C., Kupka, T., Schmeer, R., Meyenburg, I. \& Marschollek, M. (2015). Developing a mobile application for recording learning experiences in nursing practice. Studies in Health Technology and Informatics, 210, 899-903.

- Brault, I., Therriault, P., St, L., y Lebel, P. (2015). Implementation of interprofessional learning activities in a professional practicum: The emerging role of technology. Journal of Interprofessional Care, 29(6), 5305.

- Brown, R., (2015). Thinking of Serving Nursing Abroad: How Technology Assists Nurses on Mission Trips. Nursing Clinics of North America, 50 (2), 399-410.

- Dragon, N., (2006). Patient care in a technological age. Australian Nursing Journal, 14(1), 16-19.

- Escudero, B., (2003). Humanismo y tecnología en los cuidados de enfermería desde la perspectiva docente. Enfermería Clínica, 13(3), 164-170. 
- Farrar, F. (2015). Transforming Home Health Nursing with Telehealth Technology. Nursing Clinics of North America, 50 (2), 269-281.

- Feito, G. (2005). La ética del cuidado: Modas, paradojas y talantes. En M. Reyes, Rivas Flores FJ, Buisán Pelay R y García Férez J, (eds.). La bioética, un puente inacabado (pp.79-87). Madrid: Asociación de Bioética Fundamental y Clínica.

- Haghenbeck, K. (2005). Critical care nurses'experiences when technology malfunctions. Journal of the New York Nurses Association, 36(1), 13-19.

- Hallmark, B. (2015). Faculty Development in simulation education. Nursing Clinics of North America, 50(2), 389397.

- Ingadottir, T. \& Jonsdottir, H. (2006). Technological dependency the experience of using home ventilators and long-term oxygen therapy: patients' and families' perspective. Scandinavian Journal of Caring Sciences, 20(1), 18-25.

- Johansson, E., Langius, A., Engervall, P. \& Wredling, R. (2005). Patients' experience of ambulatory selfadministration of pamidronate in multiple myeloma. Cancer Nursing, 28(2), 158-65.

- Jordan, S., Philpin, S., Warring, J., Cheung. Y,. \& Williams, J. (2006). Percutaneous endoscopic gastrostomies: the burden of treatment from a patient perspective. Journal of Advanced Nursing, 56 (3), pp.270-281.

- Ketel, C. (2015). Potentials of Internet-Based Patient Engagement and Education Programs to Reduce Hospital Readmissions: A Spotlight on Need in Heart
Failure. Nursing Clinics of North America, 50(2), 283291.

- Krau, S. (2015). The Influence of Technology in Nursing Education. Nursing Clinics of North America, 50 (2), 379387.

- Lilly, K., Fitzpatrick, J,. y Madigan, E. (2015). Barriers to integrating information technology content in doctor of nursing practice curricula. Journal of Professional Nursing, 31(3), 187-199.

- Mahon, M. (2006). Technology in hospice: Is it a contradiction? Home Healthcare Nurse, 24(8), 527-531.

- Marden, S. (2005). Technology dependence and healthrelated quality of life: a model. Journal of Advanced Nursing, 50(2), 187-95.

- Moore, L. y Wasson, J. (2006). An introduction to technology for patient-centered collaborative care. Journal of Ambulatory Care Management, 29(3), 195198.

- Needleman, J., Buerhaus, P., Mattke, S., Stewart, M., \& Zelevinsky, K. (2002). Nurse staffing levels and the quality of care in hospitals. The New England Journal of Medicine, 346 (22), 1715-1722.

- Sneed, R., Warren, L., Stencel, M. y Stencel, C. (2004). Policy Versus Practice: comparison of Prescribing Therapy and Durable Medical Equipment in Medical and educational Settings. Pediatrics, 114(5), 612-25.

- Tearl, D. y Hertzog, J. (2007). Home Discharge of Technology Dependent Children: Evaluation of a Respiratory- Therapist Driven Family Education Program. Respiratory care, 52 (2), 171-176.

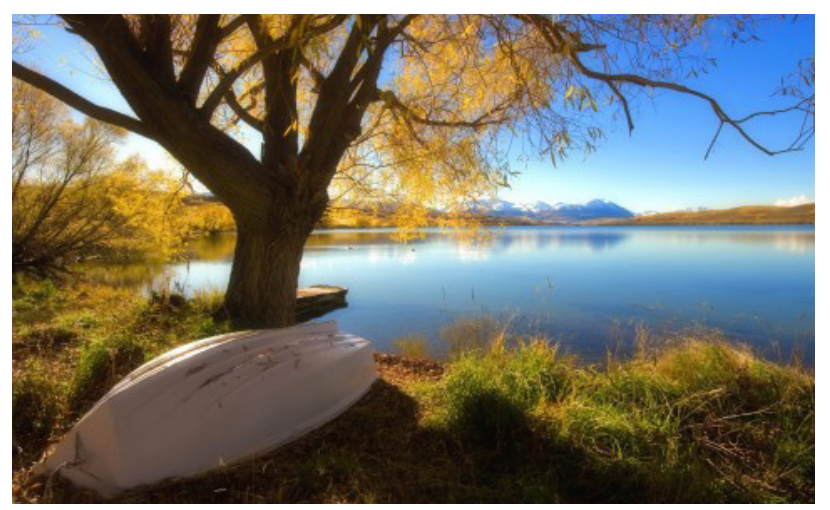

